\title{
Informed consent for epidural analgesia in labour
}

W E are frequently asked: "Can a woman in the throes of labour give consent to epidural pain relief?" One can paraphrase this question by asking: "Can a woman who has never experienced labour pain give informed consent to epidural analgesia?"

In this issue of the Journal, Jackson et al. have made an excellent contribution to this debate. ${ }^{1}$ Their survey of 60 women in labour concluded that these women, who were able to conduct a $10 \mathrm{~min}$ interview while labouring, clearly were also able to consent to the proposed epidural.

What does giving and obtaining consent mean in these circumstances?

In Canada, consent must be voluntary. Therefore no coercion must exist, and no third party can tell a competent woman to give consent. The woman must have the capacity to understand the proposed treatment, its risk, benefits and alternatives. This is where the debate has focused with regard to women who are distressed and crying out for pain relief. Do they truly understand what having an epidural means? Jackson et al. have convincingly shown that the majority of their patients' ability to understand epidural risks was not affected by labour pain, anxiety or opioid premedication. Only three were unable to complete the questions due to labour pain. The pain scores of the women taking part in the study were high but similar to those of most women before epidural analgesia takes effect. ${ }^{2}$ Therefore, even when a woman is in pain, it is reasonable in most circumstances to embark upon an explanation of the proposed procedure. It is also of interest that $46 \%$ of the women studied had received an opioid before the study. Opioids are usually given early in labour, before the pain becomes too severe, in order to give some rest. Opioids are not very good pain relievers in labour, and do not necessarily impair a woman's ability to decide that more effective pain relief is warranted. ${ }^{3}$ Individual assessment should be made and documented as to each patient's ability to appreciate the information and make a reasoned decision, including a note as to the drug, dose and time of any medication that might impair judgment.

If a woman is mentally incapable of giving consent, there are mechanisms in place for substitute decision makers to consent on her behalf. However, with few exceptions, substitute decision making has no place when the woman has not been assessed as being incapable. In the study the women were asked if it would be helpful to "have a friend help me decide", and many of them said it would. It must be understood that such "help" does not absolve a capable patient of the responsibility of making the final decision herself. Similarly, a partner has no right to refuse epidural analgesia for the woman in labour, and health care professionals should understand the patient's right and responsibility to make her own decision having been fully informed. What about age? The concept that the patient's ability to understand the information and appreciate the consequences of her decision, rather than a specific age, has been repeatedly confirmed by the courts in Canada. Capacity to consent or refuse treatment is seen as the ability to understand information that is relevant to making a decision about the proposed treatment and to appreciate the reasonably foreseeable consequences of a decision or lack of decision. ${ }^{4}$

The consenting patient must also be properly informed. Debate continues around what this means with regard to labour analgesia. Canadian courts have held that the risks and benefits of the procedure must be fully explained, as well as any alternatives and the risk of no analgesia. We tend to focus on the risks, as is proper, but we must also remember that there are considerable benefits from labour analgesia, which in a given case may be life saving for both the mother and the baby. We should, therefore, take the circumstances of the birth into consideration when explaining the proposed therapy.

From the Department of Anesthesia, McMaster University* and Department of Research and Education, Canadian Medical Protective Association. $\dagger$

Address correspondence to: Dr. Kari G. Smedstad, Department of Anesthesia, HSC-2U5, McMaster University, 1200 Main Street West, Hamilton, Ontario, Canada L8N 3Z5. Phone: 905-521-2100, Ext. 75175; Fax: 905-523-1224; E-mail: smedstad@fhs.mcmaster.ca 
In 1980, the Supreme Court of Canada held that the adequacy of the consent explanation is to be judged by what a reasonable patient in that particular patient's circumstances would have expected to hear before consenting to or refusing treatment.

We are obligated to answer any questions that the woman may ask regarding risks, and "without being questioned, disclose to him [her] the nature of the proposed operation, its gravity, any material risks and any special or unusual risks ...". 5

Jackson's study confirms that women today are very well informed about pain relief issues. Frequently, women in labour do not want to ask any further questions. While under the circumstances, this is understandable, it is still necessary to explain briefly the material risks and benefits. Generally, the more frequent the risk, the greater the obligation to discuss it. Headache, for example, as a result of unintentional dural puncture occurs with a frequency of $1 / 100$ in teaching hospitals. ${ }^{6}$ This is common, and warrants a comment in all cases. While risks, such as death, paralysis and nerve damage are exceedingly rare, such rare but serious risks are considered material and require disclosure. It may be prudent to compare the risk with something tangible, such as the risk of childbirth or driving an automobile to the hospital. Detailed explanations are not necessary unless the woman asks specific questions. Jackson et al. showed that women want to be informed about potential complications, but knowledge of potential complications would not dissuade most women from consenting to an epidural.

Discussion of risks may be best entertained before labour. Information pamphlets, which explain the procedure and the risks in simple language are now commonly used. These ideally should be distributed in the ante natal clinic, so women can read them at leisure. It is generally not necessary to mention statistics in such brochures. Brochures help to inform patients about the proposed treatment but are adjunctive to the discussion between the doctor and the patient. They do not replace the informed consent discussion. In individual cases some risks (or benefits) may be particularly relevant, and an important determinant of materiality. We should relate our consent discussion to these individual circumstances. We should then make a contemporaneous note of the discussion on the clinical record. In the real world, however, women arrive on the labour floor without the benefit of previous discussion, and it is therefore helpful to know that such discussion can take place while in labour. The paper in this journal helps us appreciate what most women want to hear and that they are capable of understanding the information.
Recent changes in pain relieving techniques may have introduced new hazards to labour analgesia. Some have expressed concern that the frequency of neurological sequelae may have increased with the introduction of spinal and combined spinal-epidural analgesia (CSE). A recent survey in England suggested an incidence of neurological sequelae of 1.14 per thousand CSEs.?

However, neurological complications also arise from pregnancy itself. ${ }^{8}$ Transient paresthesia and motor weakness after delivery has an incidence of 1.9 per 1000 deliveries.

Does the woman need to sign a consent form? Some hospitals have specific requirements to complete a form. However, the Canadian Medical Protective Association (CMPA) reminds physicians that any form is only one piece of evidence that will be considered by the courts. Consent forms will be of little benefit if a patient can convince a court that the discussions were inadequate or did not take place at all. A consent form should not obscure the important fact that the form itself is not informed consent. The dialogue with the woman, followed by a contemporaneous note in the chart about the relevant discussion is more important than a form. We caution anesthesiologists against refusing treatment for a patient who has given verbal consent on the basis of administrative delays in obtaining a signed consent form. When circumstances permit, it is advisable to obtain a signed consent form.

A recent survey in the U.S.A. suggests that women who had to sign a consent form found that written consent would help them "remember and appreciate the different anesthetic options, risks and procedures", while some thought a written consent process was "alarming".

These forms were really information pamphlets. In Canada, the CMPA advocates a simple, nonspecific form such as the one printed in the CMPA Consent guide. ${ }^{4}$

Patient autonomy is entrenched in our legal system. A woman owns her body, and has the right to consent to, or withhold consent for treatment even though it may not be in her best interests. She also has the right to change her mind. Not withstanding strong opinions before labour, and in some cases written birthing plans, a woman can always withdraw her refusal, and consent to epidural analgesia. Again, a note in the chart about this will be helpful.

We thank Jackson et al. for contributing to our understanding of the needs of women in labour.

A young anesthesiologist, who recently gave birth to her first child, told me when I attended to her request for epidural analgesia, that "I have never been so sure of anything in my life, as I am about having this epidural". She would epitomize the "reasonable, fully informed patient". 


\section{Le consentement éclairé à l'analgésie épidurale pendant le travail}

On demande souvent : «Pendant les douleurs du travail, une femme peut-elle consentir à recevoir une analgésie épidurale?» On peut paraphraser cette question en disant : «Est-ce qu'une femme qui n'a jamais vécu les douleurs de l'accouchement peut donner un consentement éclairé à l'analgésie épidurale?»

Dans le présent numéro du Journal, Jackson et coll. apportent une excellente contribution à ce débat. ${ }^{1}$ À la suite d'une enquête menée auprès de 60 femmes en travail, ils concluent que si elles ont pu participer à une interview de $10 \mathrm{~min}$ pendant le travail, elles peuvent certainement consentir à l'analgésie épidurale qu'on leur propose.

Que signifie donner et obtenir un consentement dans de telles circonstances?

Au Canada, le consentement doit être volontaire. Aucune pression ne peut être esercée et aucune tierce partie ne peut dire à une femme avertie de donner son consentement. La femme doit avoir la capacité de comprendre le traitement proposé, ses risques, ses bénéfices et les autres solutions possibles. C'est làdessus que le débat s'est concentré en regard des femmes qui souffrent et réclament avec insistance d'être soulagées. Comprennent-elles vraiment ce que signifie recevoir une analgésie épidurale? Jackson et coll. ont montré de façon convaincante que la capacité de la majorité de leurs patientes de comprendre les risques de l'analgésie épidurale n'était pas influencée par la douleur du travail, l'anxiété ou la prémédication avec des opiö̈des. Trois patientes seulement n'ont pu remplir le questionnaire à cause des douleurs. Les scores de douleurs des femmes de l'étude étaient élevés, mais similaires à ceux de la plupart des femmes, avant que l'analgésie n'ait produit son effet. ${ }^{2}$ Par conséquent, même lorsqu'une femme est en travail, on peut raisonnablement s'engager dans des explications sur la méthode d'analgésie. Il est intéressant de noter également que $46 \%$ des femmes étudiées avaient reçu un opioïde avant l'étude. Les opioïdes, habituellement administrés au début du travail, accordent un certain répit avant que les douleurs ne deviennent trop intenses. Ce ne sont pas de très bons analgésiques pendant le travail et ils ne nuisent pas nécessairement à la capacité de décider si un autre médicament plus effi- cace serait justifié. ${ }^{3}$ Une évaluation individuelle de la capacité à comprendre les informations et à prendre une décision réfléchie devrait être faite. Elle devrait inclure une note au dossier sur le médicament donné, la dose et le temps ou il a pu altérer le jugement.

Si une femme ne peut mentalement accorder son consentement, on peut utiliser les mécanismes déjà mis en place pour que des remplaçants décident à sa place. Cependant, à quelques exceptions près, personne ne peut décider à la place d'une femme qui n'a pas été jugée comme incapable de le faire. On a demandé aux femmes de l'étude si elles trouvaient utile d' 'aavoir quelqu'un pour les aider à prendre leur décision», et nombre d'entre elles ont reconnu que ce serait en effet utile. Il faut bien comprendre que cette «aide» ne réduit pas la responsabilité d'une patiente, capable de décider, de prendre elle-même la décision finale. De même, un partenaire ne peut refuser une analgésie épidurale pour une patiente en travail et les professionnels de la santé doivent connaitre le droit et la responsabilité de la patiente de prendre ses propres décisions après avoir reçu toutes les informations nécessaires. Qu'en est-il de l'âge? Dans les cours du Canada, on a confirmé à maintes reprises que la capacité d'un patient à comprendre les informations et à évaluer les conséquences de sa décision est plus importante que l'âge. La capacité de consentir à un traitement ou de le refuser est décrit comme une habileté à comprendre des informations pertinentes à la prise de décision au sujet d'un traitement proposé et à évaluer raisonnablement les conséquences prévisibles d'une décision ou d'une absence de décision. ${ }^{4}$

La patiente doit aussi être complètement informée. On discute encore de ce que cela signifie par rapport à l'analgésie pendant le travail. Les cours canadiennes ont soutenu que les risques et les avantages de la technique doivent être très bien expliqués, de même que les autres choix possibles et les risques de l'absence d'analgésie. Nous avons tendance à insister sur les risques, avec raison, mais nous devons aussi penser aux avantages considérables à l'analgésie pendant le travail qui, dans certains cas, peut sauver la vie de la mère et du bébé. Nous devons donc tenir compte des circonstances de la naissance quand nous expliquons la thérapie proposée.

En 1980, la Cour suprême du Canada a soutenu que le caractère adéquat de l'explication du consentement doit être vu comme ce qu'une patiente apte à décider s'attend à recevoir comme informations avant de consentir au traitement ou de le refuser dans les circonstances particulières où elle se trouve.

Nous avons l'obligation de répondre à toute question que la femme pourrait demander au sujet des 
risques, et «sans laisser le moindre doute, lui révéler la nature de l'opération proposée, sa gravité et tout risque important ainsi que tout risque particulier ou inhabituel...». 5

L'étude de Jackson confirme que les femmes sont aujourd'hui très bien informées sur les analgésiques. Il arrive souvent que les femmes en travail ne veuillent pas d'explications plus poussées. C'est compréhensible, dans les circonstances, mais il demeure nécessaire de fournir de brèves explications sur les avantages et inconvénients importants. Généralement, plus le risque est élevé, plus il faut en discuter. Les céphalées, par exemple, comme résultat d'une brèche durale accidentelle, surviennent selon une fréquence de 1/100 dans les hôpitaux d'enseignement. ${ }^{6}$ C'est donc fréquent et cela justifie toujours une explication. Des risques comme la mort, la paralysie et l'atteinte neurologique sont extrêmement rares, mais sérieux, et doivent donc être révélés. Par prudence, on peut les comparer avec des risques connus, comme ceux qui entourent la naissance ou le trajet en automobile pour venir à l'hôpital. Des explications détaillées ne sont pas essentielles, sauf si la patiente pose des questions spécifiques. Jackson et coll. ont montré que les femmes désiraient être informées sur les complications possibles, mais que le fait de savoir ne les a pas empêchées de consentir à une analgésie épidurale.

Une discussion sur les risques est plus profitable avant le travail. Des brochures d'informations qui expliquent le procédé et les risques en langage simple sont maintenant présentées couramment. Idéalement, on devrait les distribuer dans les cliniques prénatales pour permettre aux femmes de les lire à tête reposée. Il n'est habituellement pas nécessaire de faire état de statistiques dans ces brochures. Elles aident à informer sur le traitement proposé, mais sont accessoires à la discussion entre le médecin et sa patiente. Elles ne remplacent pas la discussion sur le consentement éclairé. Certains cas particuliers présentent des risques (ou des bénéfices) spécifiques et importants. Nous devons donc baser notre discussion du consentement sur ces circonstances individuelles. Nous devons donc inscrire une note au dossier au moment de cette rencontre. Dans les faits, les femmes arrivent à la salle de travail sans avoir participé à une discussion préalable et il est bon de savoir qu'on peut remédier à la situation pendant le travail. L'article du présent numéro nous aide à prendre conscience de ce que les femmes veulent savoir et du fait qu'elles ont la capacité de comprendre les renseignements fournis.

Les modifications récentes des techniques comportent peut-être d'autres risques à l'analgésie pendant le travail. Certains ont exprimé leurs craintes que la fréquence de séquelles neurologiques ait augmenté depuis l'arrivée de la rachianalgésie et de l'analgésie rachidienne et péridurale combinée (RPC). Une récente enquête menée en Angleterre montre une incidence de séquelles neurologiques de 1,14 sur 1000 RPC?

Toutefois, les complications neurologiques peuvent originer de la grossesse elle-même. ${ }^{8} \mathrm{La}$ paresthésie transitoire et la faiblesse musculaire qui suivent l'accouchement présentent une incidence de 1,9 sur 1000 naissances.

Les femmes doivent-elles signer une formule de consentement? Certains hôpitaux ont des exigences spécifiques pour remplir une formule. Pourtant, l'Association canadienne de protection médicale (ACPM) rappelle aux médecins que toute formule n'est qu'une preuve parmi d'autres qui pourraient être utilisées en cour. Les formules de consentement ne seront pas très utiles si une patiente peut convaincre la cour que l'explication n'a pas été complète ou n'a pas eu lieu du tout. Une formule de consentement ne doit pas cacher le fait qu'elle ne soit pas en elle-même un consentement éclairé. Le dialogue avec la patiente, suivi d'une note au dossier au sujet de la discussion, est plus important qu'une formule. Nous mettons en garde les anesthésiologistes qui voudraient refuser l'analgésie à une patiente qui a donné son assentiment verbal, sous prétexte d'un délai administratif à obtenir le consentement écrit. Quand les circonstances le permettent, il est recommandé de faire signer une formule de consentement.

Une enquête récente faite aux É-U suggère que les femmes qui devaient signer une formule de consentement ont jugé que ce geste les a aidées à «se rappeler et à évaluer les différentes options anesthésiques, les risques et les techniques», tandis que certaines trouvent que le consentement écrit a été une démarche «alarmante».?

Ces formules étaient essentiellement des brochures d'information. Au Canada, l'ACPM conseille une formule simple, non spécifique comme celle qui apparaît dans son guide sur le consentement. ${ }^{4}$

L'autonomie de la patiente est solidement établie dans notre système légal. Le corps d'une femme lui appartient; elle a le droit d'accorder ou de refuser son consentement à un traitement même si ce n'est peutêtre pas dans son meilleur intérêt. Elle a aussi le droit de changer d'idée. En dépit d'opinions fermes arrêtées avant le travail et, dans certains cas, malgré un plan prévu sur papier, une femme peut toujours annuler son refus et consentir à l'analgésie épidurale. Ici encore, une note au dossier à ce sujet sera utile.

Nous devons reconnaître la contribution de Jackson et coll. à la compréhension des besoins des patientes en travail obstétrical. 
Une jeune anesthésiologiste, qui a récemment donné naissance à son premier enfant, me disait au moment où je répondais à sa demande d'une analgésie épidurale : «De ma vie, je n'ai jamais été aussi sûre d'une chose, d'accepter cette épidurale». Elle représentait la «patiente compétente et bien informée».

\section{References}

l Jackson A, Henry R, Avery N, VanDenKerkhof E, Milne B. Informed consent for labour epidurals: what labouring women want to know. Can J Anesth 2000; 47: 1068-73.

2 Smedstad KG, Morison DH. A comparative study of continuous and intermittent epidural analgesia for labour and delivery. Can J Anaesth 1988; 35: 234-41.

3 Paech MJ. The King Edward Memorial Hospital 1000 mother survey of methods of pain relief in labour. Anaesth Intensive Care 1991; 19: 393-9.

4 Canadian Medical Protective Association. Consent: A Guide for Canadian Physicians, 3rd ed. Ottawa, Canada. 1996.

5 Reibl vs Hughes 1980; 2. S.C.C. 880.

6 Smedstad KG. Dealing with post-dural puncture headache - is it different in obstetrics? (Editorial) Can J Anaesth 1998; 45: 6-9.

7 Holloway J, Seed PT, O'Sullivan G, Reynolds F. Paraesthesiae and nerve damage following combined spinal epidural and spinal anaesthesia: a pilot survey. Int J Obstet Anesth 2000; 9: 151-5.

8 Holdcroft A, Gibberd FB, Hargrove RL, Hawkins DF, Dellaportas CI. Neurological complications associated with pregnancy. Br J Anaesth 1995; 75: 522-6.

9 Gerancher JC, Grice SC, Dewan DM, Eisenach J. An evaluation of informed consent prior to epidural analgesia for labor and delivery. Int J Obstet Anesth 2000; 9: 168-73 